\title{
POSIBLE MODULACIÓN DE LAS CIRCULACIONES ANTICICLÓNICAS POR LAS VARIACIONES DE LA ACTIVIDAD SOLAR
}

\author{
Alejandro Lomas González ${ }^{(1)}$, Ricardo Torrijo Murciano ${ }^{(2)}$. \\ (1) AEMET, Leonardo Prieto Castro 8, alomasg@aemet.es \\ (2) AEMET, Leonardo Prieto Castro 8, rtorrijom@aemet.es
}

Uno de los aspectos menos estudiados y con clara conexión con la variabilidad natural del Sistema Climático son los asociados a los cambios de circulación atmosférica con su concatenación de causas y efectos que podrían tener un efecto final en el balance radiativo del planeta. También se suele postular que esos cambios pueden deberse al factor antropogénico aunque se debate en qué medida entran en juego tanto este como la propia variabilidad.

Los anticiclones subtropicales son los grandes y principales engranajes de la meteorología tropical y a su vez están en contacto con la circulación de latitudes medias formando parte de la transmisión de energía y momento. Los trópicos abarcan prácticamente la mitad de la superficie terrestre, están situados en su mayor parte sobre superficie marina y reciben la mayor parte de la energía que llega del Sol por lo que son candidatos ideales para investigar una posible influencia de la variabilidad solar en su clima y por extensión en el clima de todo el planeta. A ellos hay que unir el efecto modulador o amplificador del ENSO.

Se conoce la influencia del ciclo solar en distintas variables meteorológicas pero no se ha podido determinar el mecanismo físico que justifique con exactitud los efectos finales que muestra la Estadística. En este trabajo, además de sopesar aproximaciones ya elaboradas, se planteará una hipótesis que indaga en las posibles influencias de las variaciones de la actividad solar, por un lado en el desarrollo del fenómeno ENSO y por otro en la modificación del gradiente vertical de temperatura en la troposfera tropical. Las repercusiones de esa modificación pueden afectar a todas las distintas partes de la célula de Hadley, especialmente a los alisios, hecho que puede modificar no solo el balance energético de la Tierra sino también cambiar los patrones meteorológicos de la circulación general.

Las conclusiones de la investigación estarán sometidas, por su valor predictivo, a las observaciones de las variables meteorológicas en el futuro, que las reforzarán o descartarán.

Haigh et al (2005) establecieron que la estratosfera juega un papel en modular el impacto de la actividad solar en el clima. En momentos de máxima actividad solar se comprueba que se produce un calentamiento estadísticamente significativo en la baja estratosfera de las bajas latitudes. Cuando el sol es más activo se produce un debilitamiento de los chorros así como su desplazamiento hacia los polos, además de un debilitamiento y expansión de la célula de Hadley. El reanálisis NCEP/NCAR es capaz de detectar estos patrones por medio de los datos de velocidad vertical. Calentar la baja estratosfera incrementa la estabilidad estática, baja la tropopausa. Esto conlleva cambios en toda la troposfera. 
Además reconstrucciones de la actividad ENSO de los últimos siglos (Gergis et al., 2006) han determinado la inusual naturaleza del fenómeno durante el siglo XX como período pico de las fases cálidas de El Niño que coincide también con un siglo de inusual actividad solar.

El debilitamiento de la circulación de las células anticiclónicas puede también tener otras consecuencias como la de influir en el balance radiativo del planeta como consecuencia de un aumento de las nubes bajas. La persistencia de nubes bajas en los subtrópicos está ligada a la fortaleza de la inversión de subsidencia, determinada ésta por la estabilidad de la baja tropósfera en esa zona. (Stephen et al 1993).

Los datos proxy tropicales respecto al máximo de la última edad de hielo apunta a que la línea de congelación en las montañas estaba unos 800 m más abajo (CLIMAP. 1981,4). Utilizado el gradiente vertical de temperatura normal en los trópicos esto no es coherente con la temperatura en la superficie. Esto presupone que el gradiente vertical sería más pronunciado en condiciones de frío. Lo mismo pasa con el pico de enfriamiento de la Pequeña Edad de Hielo en el siglo XVII.

\section{Introducción}

\section{A) Hechos geográficos de la zona tropical}

* La zona intertropical abarca prácticamente la mitad de la superficie terrestre

* Los rasgos más destacados de la circulación atmosférica son los anticiclones y la zonas de convergencia intertropical (ITCZ)

* La superficie es básicamente oceánica por lo que las interacciones atmósfera/océano y los intercambios energéticos son máximos

* Es la zona de entrada en la Tierra de más energía vía solar

*Es un hecho significativo que el mínimo relativo solar de los años setenta coincide con un máximo de los vientos alisios

\section{B) Variabilidad de la actividad solar}

En el trabajo clásico de Eddy de 1977 (Eddy, 1976) se especuló sobre las posibles conexiones entre la actividad solar y el clima por medio de la huella dejada por la producción de carbono 14 a lo largo de la historia. A mayor actividad solar menos producción de carbono 14 en la alta atmósfera por los rayos cósmicos que bombardean la tierra. En los trabajos de Lamb (Lamb, 1977) se empieza a hablar de períodos históricos con clima cálido o frío que parecen coincidir con períodos de mayor o menor actividad solar.

Cualquier estudio de la influencia de la variabilidad solar en el clima de la Tierra requiere tener en cuenta la variabilidad solar en el corto y largo plazo, las interacciones sol-tierra y los mecanismos que determinan la respuesta del Sistema climático a esas interacciones.

Los cambios en la irradiancia total solar que se han podido medir no parecen ser suficientes para justificar cambios importantes. El cambio de la irradiancia conocida en un ciclo solar es de $1 \mathrm{~W} / \mathrm{m}^{2}$ sobre un valor de 1366 . El efecto en el tope de la atmósfera es de $0,17 \mathrm{~W} / \mathrm{m}^{2}$, un $0,07 \%$. El cambio a largo plazo también debe ser tenido en cuenta. La observación por satélite de los últimos 30 años también muestra valores muy pequeños de unos $0,017 \mathrm{~W} / \mathrm{m}^{2}$. Usando valores de forzamiento habituales nos daría una variación de 0,07 K (Gray et al., 2010). Otro hecho significativo es que, según Solanki and Krivova 
(2004), el Sol está en un máximo de gran actividad que empezó en torno a 1920. También se sugiere que el Sol puede haber dejado el gran máximo del siglo y que no está entrando en otro gran mínimo.

Hay incertidumbre también en cuanto a la variabilidad que acompaña los cambios en la irradiancia solar total (Ermolli et al., 2013). Recientes medidas de satélite con Spectral Irradiance Monitor (SIM) sobre el satélite de radiación solar y experimento climático muestra que la variabilidad en el ultravioleta sobre la parte descendente del ciclo solar 23 puede ser considerablemente mayor que lo indicado por anteriores estimaciones (Harder et al., 2009 y Haigh et al., 2010).

Se ha intentado desde siempre establecer una conexión entre el Sol y el clima aunque ha fallado porque no se ha podido establecer una relación causal que conecte cuantitativamente los valores conocidos de la radiación solar con cambios significativos del Clima. La comparación con los forzamientos radiativos parece descartar influencias importantes de los cambios de actividad solar.

\section{C) Efectos de la variabilidad de la actividad solar en la estratosfera}

El ozono es el principal gas involucrado en el calentamiento radiativo estratosférico. Las variaciones de velocidad de producción de ozono son debidas a los cambios de radiación en UV (Haigh, 1994). Aunque la absorción del ultravioleta es solo una pequeña proporción de la energía solar entrante, dentro del ciclo solar puede haber variaciones de entre 2 y $4 \%$ de ozono en el espectro entre $240-320 \mathrm{~nm}$ donde prevalece la absorción por ozono y de hasta el 6\% están presente cerca de los $200 \mathrm{~nm}$ donde se produce la disociación del oxígeno y la formación de ozono. Estos valores deben entonces compararse con las variaciones de tan solo el $0,07 \%$ de la irradiancia solar total. Además hay pruebas estadísticamente significativas para una variación de 11 años en la temperatura estratosférica y en los vientos zonales atribuibles a la radiación UV (Frame and Gray, 2010). También las variaciones se pueden producir a través de cambios en el transporte por efectos indirectos en la circulación (Shindell et al., 2006).

En conclusión, las variaciones de la irradiancia solar en el lejano UV (120 a $200 \mathrm{~nm})$ y en el medio UV (200 a $300 \mathrm{~nm}$ ) (UV) son los factores primarios para el calentamiento, composición y cambios dinámicos de la estratosfera y aunque componen una pequeña porción de la radiación entrante muestra relativamente grandes variaciones entre el máximo y el mínimo de la actividad solar en comparación a los correspondientes cambios de la irradiancia total.

Considerando que el calentamiento de la estratosfera durante el ciclo solar tiene el potencial de influir a la troposfera indirectamente, por medio del acoplamiento dinámico, y por esto al clima (Haigh, 1996), el UV puede tener un más significativo impacto en el clima que los cambios que tan solo la irradiancia total podría sugerir. La medida de UV presenta varios aspectos controvertidos. Medidas hechas en el espacio los últimos 30 años indican que las variaciones en el UV dan cuentan de un 30\% de las variaciones dentro de un ciclo solar No obstante, se estima que la contribución del UV por debajo de los $400 \mathrm{~nm}$ es del 30 al $90 \%$ de la variación correspondiente a la irradiancia total, con un valor más probable del 60\% (Rottman et al., 2006). 
Haigh et al (2005) usaron un análisis de regresión múltiple de datos del reanálisis NCEP/NCAR para mostrar una respuesta correspondiente a un aumento de la actividad solar que provoca un debilitamiento y desvío hacia los polos de los chorros subtropicales, con el efecto transmitido verticalmente a la troposfera tanto en lo que respecta a la temperatura como a los vientos zonales. Esta señal es separable de otras influencias como las del ENSO y la NAO, y es similar a los resultados de previos estudios que usaban modelos climáticos globales en los que se inclúa el efecto de la variación del ciclo solar de 11 años de la radiación UV.

Los mismos autores señalan que experimentos realizados con modelos climáticos globales simplificados muestran que calentamientos genéricos de la baja estratosfera tienden a debilitar los chorros subtropicales y las circulaciones meridianas medias troposféricas. El calentamiento de la parte baja de la estratosfera incrementa la estabilidad estática, hace descender la tropopausa. De forma solidaria cambian la localización e intensidad de la corriente en chorro, la trayectoria de las tempestades y la circulación meridiana inducida por las borrascas.

Los patrones exactos de respuesta dependen de la distribución de las perturbaciones del calentamiento de la estratosfera: el calentamiento en las latitudes medias y altas hace que los chorros se muevan hacia el ecuador y se debilitan las células de Hadley mientras que el de las bajas latitudes produce un cambio de los chorros hacia el polo y la expansión de las células de Hadley. También produce un debilitamiento de los chorros subtropicales. Los resultados de los experimentos del calentamiento en altas/ bajas latitudes son cualitativamente similares a las respuestas encontradas en los datos observacionales relativos a la mayor actividad solar y a las simulaciones con modelos de circulación global de un aumento de la radiación UV.

El estudio se hizo con datos desde 1979 debido a la dudosa calidad de los datos de la estratosfera tropical previa a la existencia de satélites .

\section{D) Posibles efectos de la variabilidad solar en el fenómeno ENSO}

El Niño Oscilación del Sur (ENSO) es un patrón dominante de la oscilación natural del Sistema acoplado atmósfera/océano en el océano Pacífico. La cuestión de si el Pacífico Ecuatorial responde al calor radiativo en la forma de El Niño o La Niña es muy debatido en el contexto del hipotético calentamiento global. Hay dos teorías contrapuestas que compiten. Clement et al. (1996) afirman que la temperatura de la superficie marina en el Pacifico oriental es controlada por el agua fría generada por emersión y de esta forma el calentamiento incrementa solo la temperatura del mar en el Pacífico Oeste, lo que produce un reforzamiento de la circulación de Walker, es decir una respuesta de tipo La Niña. Held and Soden (2006) y Gabriel et al. (2007) sugirieron que las circulaciones tropicales se debilitarían en un clima más cálido. El debilitamiento de los alisios llevaría a una circulación tipo El Niño. Este esquema está apuntalado por los modelos acoplados atmósfera-océano.

Los datos proxy tropicales respecto al máximo de la última edad de hielo apuntan a que la línea de congelación en las montañas estaba unos $800 \mathrm{~m}$ más abajo (Gray et al., 2010). Utilizando el gradiente vertical de temperatura normal en los trópicos esto no es coherente con la temperatura en la superficie. Esto presupone que el gradiente vertical 
sería más pronunciado en condiciones de frío. Lo mismo pasa con el pico de enfriamiento de la Pequeña Edad de Hielo en el siglo XVII.

\section{E) Variaciones históricas recientes del fenómeno ENSO}

Gergis y Fowler (2006) reconstruyeron desde el siglo XVI los fenómenos ENSO. Demostraron la inusual naturaleza de ENSO durante el siglo XX. La máxima actividad de La Niña tuvo lugar durante los siglos XVI y XIX, (años de poca actividad solar y Pequeña Edad de Hielo) mientras que el siglo XX es identificado como el período pico de la actividad El Niño. 55\% de los El Niño extremos en todos los años reconstruidos desde 1525 ocurrieron en el siglo XX. Aunque eventos extremos de ENSO ocurrieron también durante los 478 años reconstruidos, aproximadamente el $43 \%$ de los extremos y $28 \%$ de los que tuvieron oscilaciones más marcadas fueron en el siglo XX. En particular, el período post 1940 da cuenta del 30\% de los extremos años ENSO.

Esta tendencia hacia más actividad ENSO se apuntó en el cuarto informe del IPCC. Trenberth et al. (2007) observaron cómo desde el cambio 1976-1977 a temperaturas oceánicas por encima de lo normal en el Pacífico central y ecuatorial, ha habido una tendencia hacia más largos y fuertes eventos El Niño. Por otro lado se sabe que las temperaturas medias globales están influenciadas por ENSO por medio de grandes intercambios de calor entre el océano y la atmósfera.

\section{Método y resultados}

El trabajo que hemos desarrollado se ha elaborado en las siguientes fases:

1. Se investigan los cambios de las circulaciones anticiclónicas subtropicales inducidos por la variación solar en las zonas más alejadas de las oscilaciones monzónicas y de las zonas continentales: el Pacífico central.

2. Se utilizó el reanálisis NCEP/NCAR (Kalnay et al., 1996) para analizar las relaciones y correlaciones entre el flujo solar, las temperaturas de la alta troposfera tropical, el viento zonal tropical ( $1000 \mathrm{hPa}$ ) y las temperaturas de la superficie del mar en los trópicos. También se investigaron los posibles cambios de la circulación atmosférica en esa zona y su posible conexión con los cambios del gradiente vertical de temperatura de la alta troposfera inducidos hipotéticamente por la mayor o menor actividad solar.

3. En una primera fase se localizó una zona ecuatorial pacífica cercana a la del El Niño 3.4. Se busca una correlación entre la temperatura de la superficie marina con el flujo solar F10.7.

4. Establecida la hipótesis de que la mayor o menor actividad solar modifica el perfil de temperatura vertical en la zona tropical $\left(20^{\circ} \mathrm{N}-20^{\circ} \mathrm{S}\right)$ y por ello la estabilidad estática se ha buscado un predictor que pueda anticipar la mayor o menor actividad de las células anticiclónicas.

5. Finalmente como hipótesis se plantea un modelo de reforzamiento de la circulación anticiclónica que puede tener una repercusión en el balance radiativo del planeta por medio de las nubes bajas subtropicales.

Utilizando el reanálisis se encuentra (Fig.1) que la temperatura en niveles altos de la troposfera tropical, en $200 \mathrm{hPa}$, está correlacionada tanto con la temperatura de la superficie del mar (en este caso he usado el índice Niño3,4) y también simétricamente con 
la actividad solar. Dado que se conoce la influencia de la variabilidad del ciclo solar en las temperaturas de la baja estratosfera tropical es evidente que se puede investigar qué efecto en la estabilidad estática pueden ejercer ambos efectos superpuestos también en la alta troposfera. Cabe pensar que si actúan sincrónicamente tanto el efecto desde arriba, la mayor radiación solar, especialmente el ultravioleta, y el efecto desde abajo, el aumento de la temperatura de la superficie marina, se produciría una realimentación que modificaría sensiblemente el gradiente vertical de temperatura y la estabilidad estática.
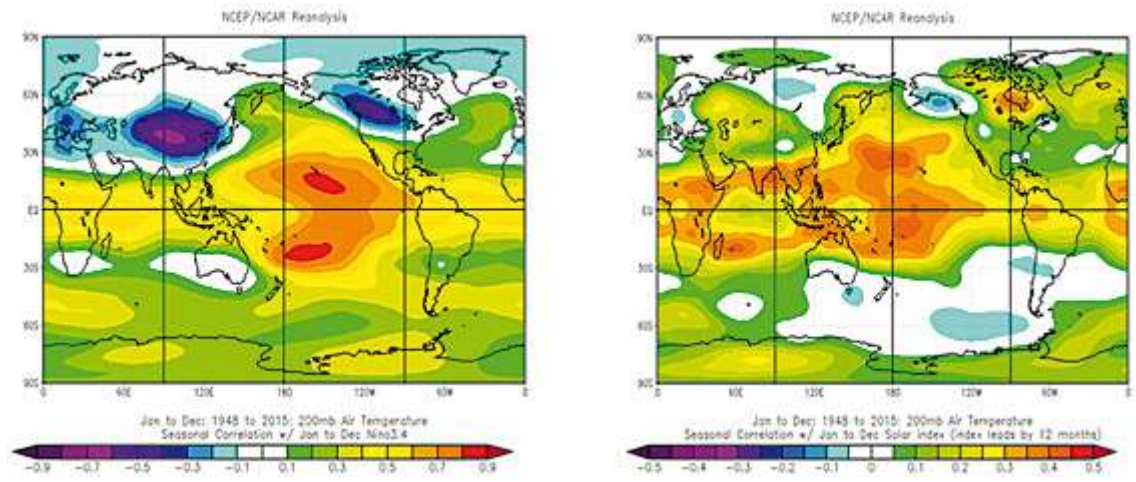

Fig. 1 Correlación de la temperatura en $200 \mathrm{hPa}$ con la temperatura de la superficie del mar en la zona 3,4 de El Niño (izquierda) y con el flujo solar de $10,7 \mathrm{~cm}$. Fuente: Reanálisis tomado de NOAA/ OAR/ESRL PSD, Boulder, Colorado, USA, de su página web en http://www.esrl.noaa.gov/psd/

Para buscar la posible relación entre los distintos niveles del ciclo solar y la temperatura de mar se ha buscado la zona más propicia en la zona del Pacífico donde el fenómeno ENSO alcanza su mayor desarrollo: $160-250$ E, $5^{\circ} \mathrm{N} / 5^{\circ} \mathrm{S}$. Las variables de la Figura 2 son el flujo solar de $10,7 \mathrm{~cm}$, buen proxy para la actividad solar, y la temperatura media anual de la superficie del mar pero destencionalizada. He superpuesto (barras negras) los momentos de significativas erupciones volcánicas que parecen también afectar al desarrollo de los ENSO. El flujo solar se representa desplazado hacia delante un año.

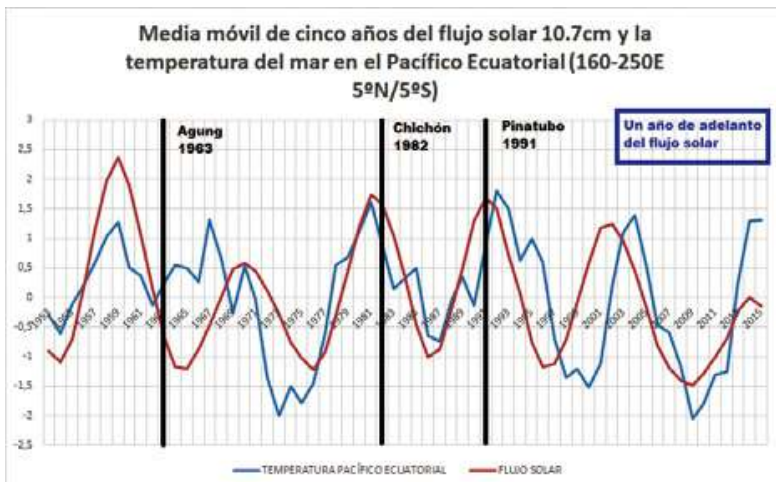

He aplicado a ambas la media móvil de 5 años para conseguir cierta coherencia. Hay datos de reanálisis desde 1948.

Fig .2 Medias móviles 5 años del flujo solar de $10,7 \mathrm{~cm}$ y la temperatura del agua del mar destencionalizada entre 160-250E y $5^{\circ} \mathrm{N}-5^{\circ} \mathrm{S}$. Se adelanta un año el flujo solar. Fuente: Reanálisis tomado de NOAA/OAR/ESRL PSD, Boulder, Colorado, USA, de su página web en http://www.esrl.noaa.gov/psd/ 
Hay que tener en cuenta que los fenómenos ENSO tienden a amplificarse en un sentido u otro por lo que la explicación de la posible correlación tiene que ver con que hay más probabilidades de que se forme un episodio cálido/frío El Niño/La Niña con mayor/menor actividad solar. De la observación de la figura 2 cabe conjeturar que en las proximidades de un máximo/mínimo solar hay mayores probabilidad de un El Niño/La Niña.

Los fenómenos de El Niño están asociados en su comienzo a la disminución de los vientos zonales tropicales (alisios) y el acoplamiento océano/atmósfera conforma el juego de circulaciones atmosféricas. De forma que toda la circulación tropical, que hay que contemplar como un todo que se mueve en las tres dimensiones (Hadley y Walker), acaba condicionada por los distintos patrones de cada episodio, especialmente en el Pacífico. Se parte de la hipótesis de que el comienzo de cada episodio puede venir de diferencias en la estabilidad estática de la atmosfera tropical. La mayor/menor estabilidad estática favorecerá un debilitamiento/reforzamiento de las corrientes ascendentes convectivas en las ramas ascendentes de la célula de Hadley. Esto afectará a los movimientos de toda ella, influyendo en el debilitamiento/reforzamiento de la velocidad de los alisios favoreciendo por su acción el aumento/disminución de la SST. Todo ello es coherente con lo detectado en cuanto al debilitamiento de las circulaciones de Hadley que es acompañado por un movimiento de expansión en latitud.

En conclusión hemos analizado la relación entre por un lado las diferencias de temperatura entre $500 \mathrm{hPa}$ y la tropopausa en la zona tropical $\left(10^{\circ} \mathrm{N} / 10^{\circ} \mathrm{S}\right)$ y por otro la intensidad de los vientos alisios en determinadas zonas elegidas. Para evitar las distorsiones de las circulaciones monzónicas del hemisferio norte nos hemos limitado a zonas del HS donde predomina el viento alisio además de ser la zona del planeta con menos tierra emergida. En lo que respecta a los datos del reanálisis se ha comprobado que en las zonas tropicales, especialmente en los niveles altos se ha de tener en cuenta que la calidad de los datos mejora notablemente a partir de los satélites, 1979.

Aunque se pueden elegir zonas del Pacífico cercanas a las del desarrollo de los fenómenos de El Niño hemos seleccionado contrastar la diferencia de temperatura entre la tropopausa y $500 \mathrm{hPa}$ con el viento zonal en $1000 \mathrm{hPa}$ entre el Ecuador y los $30^{\circ} \mathrm{S}$ a lo

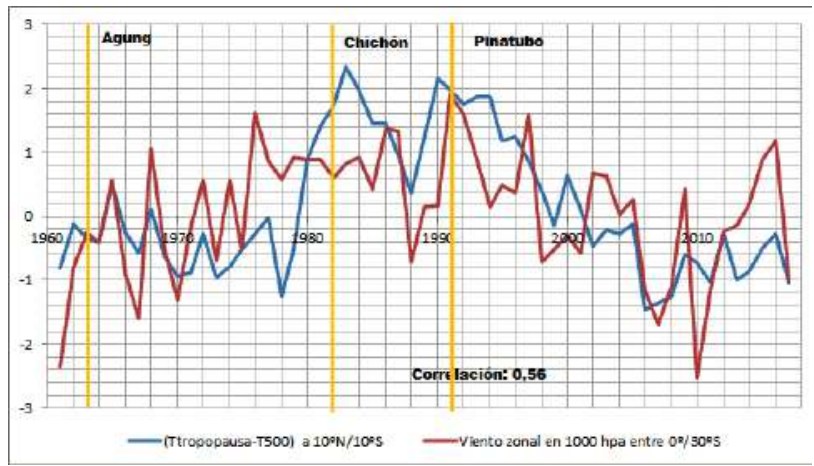

Fig. 3: Medias anuales normalizadas de las diferencias de temperatura entre 500 hPa y la tropopausa entre $\operatorname{los} 10^{\circ} \mathrm{N}$ y $\operatorname{los} 10^{\circ} \mathrm{S}$ (adelantadas un año) y por otro lado viento zonal en $1000 \mathrm{hpa}$ entre el Ecuador y los $30^{\circ} \mathrm{S}$. Fuente: Reanálisis tomado de NOAA/OAR/ESRL PSD, Boulder, Colorado, USA, de su página web en http:// www.esrl.noaa.gov/psd/ 
largo de todo el globo. En la Fig 3 hay que tener en cuenta que los valores normalizados significan: para los alisios, valores negativos vientos alisios más fuertes y para la diferencia de temperatura los valores negativos mayores diferencias que implican menos estabilidad estática. Se representa con un año de adelanto de la diferencia de temperatura con respecto al viento zonal. He vuelto a señalar las erupciones principales porque tienen un efecto en el corte de temperaturas de la estratosfera.

Como se ve la correlación es de 0,56 y como en todo este tipo de variables los años ochenta y noventa del pasado siglo se presentan como una singularidad que además coincide con la presencia de dos importantes erupciones en plenos máximos solares lo que añade más complejidad.

\section{CONCLUSIONES}

1. Las diferentes variaciones en la actividad solar dentro de sus ciclos de 11 años afectan a las temperaturas en la estratosfera y alta troposfera. Pese a la incertidumbre de no conocer con precisión la cantidad de UV que emite el Sol y que alcanza esas zonas cabe colegir que puede haber mayores diferencias que las esperables dentro y entre cada ciclo solar.

2. Con meses de retraso el efecto de esa mayor/menor radiación solar supone en las zonas de mayor incidencia, que son las tropicales, menor/mayor estabilidad estática en la parte alta de la troposfera. El incremento de la inestabilidad supone un reforzamiento en la vertical de la rama ascendente de la célula anticiclónica que incidirá en el resto de los movimientos de la misma.

3. El aumento/disminución de la corriente ascendente supondrá aumento/disminución de los vientos alisios y desactivan/activan el comienzo de los episodios de El Niño.

4. Todo esto es coherente con el debilitamiento y desvío hacia los polos de los chorros subtropicales, debilitamiento y expansión de las células tropicales de Hadley y un desplazamiento a latitudes más altas de las de Ferrell con alta actividad solar y que explicamos más arriba.

5. La zona subsidente de las células anticiclónicas generan una gran cantidad de nubes bajas asociadas a la parte inferior de la inversión de dichas altas presiones. Se sabe que a mayor estabilidad estática en esa zona mayor cantidad de nubes bajas se forman, generando un importante efecto de enfriamiento positivo (menor entrada de radiación solar) cuando eso ocurre. Se puede especular que este efecto puede ser el amplificador de un efecto solar sobre el Sistema Climático.

6. En la Fig 4 he bosquejado lo que podría ser un resumen de cómo una disminución de energía solar UV puede generar corrientes ascendentes y descendentes más potentes pero con contracción del área de influencia de las zonas tropicales.

7. Las pasadas décadas se han caracterizado por un período de relativamente alta actividad solar. El reciente débil mínimo solar y el también débil ciclo 24 parecen sugerir que ese período de alta actividad puede estar tocando a su fin. Esta circunstancia puede ser una buena piedra de toque para observar los cambios inducidos en el Sistema Climático aunque la variabilidad natural esté siempre matizada por la influencia del hipotético calentamiento global antropogénico. 


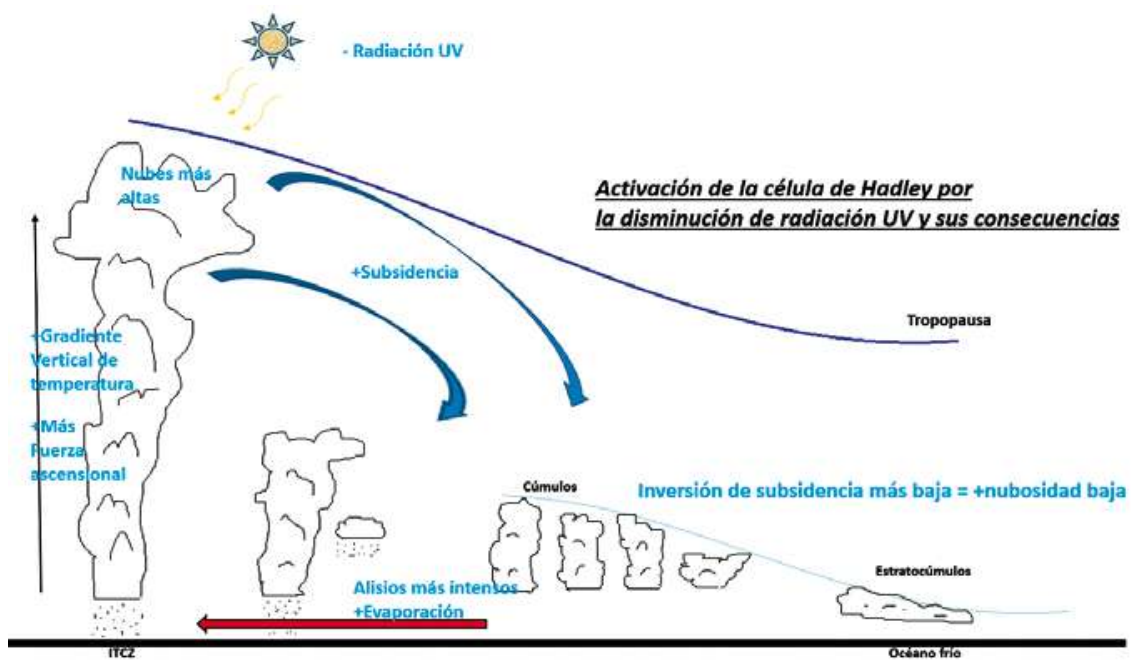

Fig. 4. Esquema propuesto de las interacciones dentro de la célula de Hadley en condiciones de disminución de radiación UV

\section{Referencias}

- Clement, A.C., R. Seager, M.A. Cane, S.E. Zebiak, 1996: An Ocean Dynamical Thermostat. J. Climate, 9, 2190-2196.

- CLIMAP Project Members, 1981: Seasonal reconstruction of the earths surface at the last glacial maximum. Geological Society of America, Map and Chart Series, 36, 18 pp.

- Eddy J.A., 1976: The Maunder Minimum. Science. 192 (4245): 1189-1202,.

- Ermolli, I., K. Matthes, T. Dudok de Wit, N. A. Krivova, K. Tourpali, M. Weber, Y. C. Unruh, L. Gray, U. Langematz, P. Pilewskie, E. Rozanov, W. Schmutz, A. Shapiro, S. K. Solanki, T. N. Woods, 2013: Recent variability of the solar spectral irradiance and its impact on climate modelling, Atmos. Chem. Phys., 13, 3945-3977.

- Frame, T.H. and L.J. Gray, 2010: The 11-Yr Solar Cycle in ERA-40 Data: An Update to 2008. J. Climate, 23, 2213-2222.

- Gabriel A. V., and B.J. Soden. 2007: Global Warming and the Weakening of the Tropical Circulation. Journal of Climate 20(17): 4316-4340.

- Gergis, J. , K. Braganza, A. Fowler, S. Mooney and J. Risbey, 2006: Reconstructing El NiñoSouthern Oscillation (ENSO) fron high resolution paleoarchives. J. Quaternary Sci., 21: 707-722.

- Gray, L. J., J. Beer, M. Geller, J. D. Haigh, M. Lockwood, K. Matthes, U. Cubasch, D. Fleitmann, G. Harrison, L. Hood, J. Luterbacher, G. A. Meehl, D. Shindell, B. van Geel and W. White, 2010: Solar influences on climate, Rev. Geophys, 48, RG4001.

- Haigh, J.D., 1994: The role of stratospheric ozone in modulating the solar radiative forcing of climate. Nature, volume 370, pages 544-546 (18 August 1994).

- Haigh, J.D., 1996: The Impact of Solar Variability on Climate. Science 17 May 1996: Vol. 272, Issue 5264, pp. 981-984. 
- Haigh, J.D., M. Blackburn, and R. Day, 2005: The Response of Tropospheric Circulation to Perturbations in Lower-Stratospheric Temperature. J. Climate, 18, 3672-3685.

- Haigh, J. D., Winning, A., Toumi, R. and J.W. Harder, 2010: An influence of solar spectral variations on radiative forcing of climate. Nature 467.

- Harder, J. W., Fontenla, J. M., Pilewskie, P., Richard, E. C. and T.N. Woods, 2009: Trends in solar spectral irradiance variability in the visible and infrared. Geophys. Res. Lett. 36, L07801.

- Kalnay et al (1996).,The NCEP/NCAR 40-year reanalysis project, Bull. Amer. Meteor. Soc., 77, 437-470.

- Kiyotaha Shibata K., K. Kodera, 2005: Simulation of radiative and dynamical responses of the middle atmosphere to the 11-year solar cycle. Journal of atmospheric and Solar-Terrestrial Physics. Vol.67. Issues 1-2, January 2005. Pages 125-143.

- Klein, S.A. and D.L. Hartmann, 1993: The Seasonal Cycle of Low Stratiform Clouds. J. Climate, 6, 1587-1606.

- Lamb, H.H., 1985 (c1977), Climate History and the future. Princeton University Press, 835pp.

- Rottman, Gary J., Thomas N. Woods, and William McClintock (2006): SORCE Solar UV Irradiance Results, Adv. Space Res., 37, 2, 201-208, 35th Committee on Space Research (COSPAR) Proceedings, Paris, France, July 18-25, 2004.

- Solanki S.K. and N.A. Krivova, 2004: Solar Irradiance Variations: From Current Measurements to Long-Term Estimates. Solar Physics (2004) 224, 197-208.

- Schmidt, G.A., J.H. Jungclaus, C.M. Ammann, E. Bard, P. Braconnot, T.J. Crowley, G. Delaygue, F. Joos,. N.A. Krivova, R. Muscheler, B.L. Otto-Bliesner, J. Pongratz, D.T. Shindell, S.K. Solanki, F. Steinhilber, and L.E.A. Vieira, 2012: Climate forcing reconstructions for use in PMIP simulations of the Last Millennium (v1.1). Geo-scientif. Model Dev., 5, 185-191.

- Shindell, D., G. Faluvegi, A. Lacis, J. Hansen, R. Ruedy, and E. Aguilar, 2006: Role of tropospheric ozone in-creases in 20th century climate change. J. Geophys. Res., 111, D08302.

- S.A. Klein and D. L. Hartmann,1993: The Seasonal Cycle of Low Stratiform Clouds. Journal of Climate 6(8), 1587-1606.

- Trenberth, K.E., P.D. Jones, P. Ambenje, R. Bojariu, D. Easterling, A. Klein Tank, D. Parker, F. Rahimzadeh, J.A. Renwick, M. Rusticucci,. B. Soden and P. Zhai, 2007: Observations: Surface and atmospheric climate change. Chapter 3 in S. Solomon, D. Qin, M. Manning, Z. Chen, M. Marquis, K.B. Averyt, M. Tignor and H.L. Miller (eds.), 2007, Climate Change 2007: The Physical Science Basis. Contribution of Working Group I to the Fourth Assessment Report of the Intergovernmental Panel on Climate Change. Cambridge University Press, Cambridge, United Kingdom and New York, NY, USA. (Contributing author: Mote), 235-336. 